\title{
Propuesta de modelo tecnológico para Flipped Classroom (T-FliC) en educación superior
}

\author{
Proposal of a Technology Model for Flipped Classroom (T-FliC) in Higher Education
}

\section{Proposta de um modelo tecnológico para sala de aula invertida (T-FliC) no ensino superior}

\author{
Matilde Basso-Aránguiz' \\ Universidad Católica de la Santísima Concepción \\ Concepción, Chile \\ matildebasso@ucsc.cl \\ iD http://orcid.org/0000-0002-0710-0640 \\ Mario Bravo-Molina ${ }^{2}$ \\ Universidad del Bío-Bío \\ Concepción, Chile \\ mbravo@ubiobio.cl \\ (iD) http://orcid.org/0000-0003-4085-1275 \\ Antonella Castro-Riquelme ${ }^{3}$ \\ Liceo Los Andes \\ Concepción, Chile \\ acastro@magisteredu.ucsc.cl \\ (iD) https://orcid.org/0000-0002-9099-0639 \\ César Moraga-Contreras ${ }^{4}$ \\ Escuela Parroquial Domingo Savio \\ Santiago, Chile \\ cmoraga@magisteredu.ucsc.cl
}

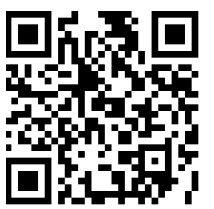

\footnotetext{
${ }^{1}$ Geóloga titulada en la Universidad de Concepción, Chile. Actualmente se desempeña como docente del Departamento de Ingeniería Civil de la Facultad de Ingeniería, Universidad Católica de la Santísima Concepción (UCSC) y es candidata al grado de Magíster en Informática Educativa y Gestión del Conocimiento. Posee publicaciones en congresos nacionales relacionados al uso de TIC en educación superior. Ha sido relatora del Taller “Evaluación y TIC" en Encuentro Latinoamericano de CDIO. Se desempeña como colaboradora en proyecto de apoyo a la docencia en la UCSC (FAD2016-11).

${ }^{2}$ Ingeniero de Ejecución en Computación e Informática. Actualmente académico del departamento de Ciencias de la Construcción de la Facultad de Arquitectura, Construcción y Diseño, Universidad del Bío-Bío, Chile y es candidato al grado de Magíster en Informática Educativa y Gestión del Conocimiento. Ponente del artículo científico"Propuesta de un Modelo de Evaluación con Uso de TIC en el Aprendizaje del Inglés en la Universidad del Bío-Bío" en el XVIII Congreso Internacional EDUTEC 2015, noviembre, Riobamba, Ecuador.

${ }^{3}$ Profesora de Lenguaje y Comunicación y candidata al grado de Magíster en Informática Educativa y Gestión del Conocimiento. Actualmente se desempeña como profesora de Lenguaje y Comunicación en el Liceo Los Andes, en la comuna de San Pedro de la Paz, Concepción, Chile.

${ }^{4}$ Profesor de Educación Física, realizando Magister en Informática Educativa y Gestión del Conocimiento, con experiencias en docencia secundaria y universitaria. Actualmente coordinador de enlaces en el Colegio Parroquial Domingo Savio. Fue profesor en Colegio Salesiano, Concepción, haciendo aportes en uso de TIC aplicados a la Educación Física y docente en la Universidad de las Américas, realizando la cátedra de Informática Educativa a la carrera de Pedagogía en Educación Física, Concepción.
} 
doi: http://dx.doi.org/10.15359/ree.22-2.2

URL: http://www.una.ac.cr/educare

CORREO: educare@una.cr

Recibido • Received • Recebido: 30 / 07 / 2016

Corregido • Revised • Revisado: 10 / 12 / 2017

Aceptado • Accepted • Aprovado: 12/ 02 / 2018

Resumen: Se presenta una propuesta de modelo tecnológico para Flipped Classroom (FC) o aula invertida, denominado T-FliC. El objetivo es dar un soporte mediado por las tecnologías de la información y comunicación (TIC) al modelo pedagógico homónimo existente, para ser considerado en futuras implementaciones en asignaturas de distintos niveles de formación en educación superior. T-FliC se basa, fundamentalmente, en la utilización de recursos tecnológicos gratuitos, teniendo como base las aplicaciones de Google (Classroom, Drive y Youtube, entre otras), debido a su uso masivo por parte de estudiantes y docentes, lo que posibilita replicar este modelo en distintos contextos educativos. T-FliC incorpora cinco fases mediadas por las TIC, que van desde la planificación de las actividades de enseñanza-aprendizaje hasta la evaluación continua de los aprendizajes. En su implementación, el modelo T-FliC considera una clase digital (aprendizaje fuera del aula) con la orientación asincrónica de personal tutor virtual, un taller que comprende el desarrollo de actividades dinámicas para el trabajo colaborativo (aprendizaje en el aula) con la guía de personal tutor presencial y un proceso evaluativo permanente y continuo mediado por herramientas tecnológicas tales como teclera, portafolio y foro que permitirá tener el registro digital de la ruta de aprendizaje del estudiantado. Este artículo incluye una revisión bibliográfica acerca del rol de las TIC en los procesos educativos y de los fundamentos de la metodología Flipped Classroom (FC). Se incorporan antecedentes de experiencias de implementación de FC en educación superior, seguido de la presentación del modelo T-FliC como una propuesta tecnológica para esta metodología. Finalmente, en las conclusiones se presentan reflexiones sobre la propuesta.

Palabras claves: Educación superior; flipped classroom; modelo tecnológico; TIC.

Abstract: The Technology Model, called T-FliC is proposed for Flipped Classroom. The aim is to provide IT facilities to the aforementioned pedagogical model. This proposal may be implemented at different levels of higher education. T-FliC is primarily based on the use of free technology resources, especially Google applications such as Classroom, Drive, and YouTube, because they are widely used by students and teachers. This extensive use permits to replicate this model in different educational contexts. The T-FliC model incorporates five ICT phases, ranging from the planning of teachinglearning activities to continuous learning assessments. The implementation of the T-FliC Model includes the following phases: a digital class (learning outside the classroom) with asynchronous guidance of a virtual tutor; a workshop involving dynamic activities for collaborative work (classroom learning) guided by a tutor in person; and an ongoing technological tools evaluation process (clickers, portfolio, and forum) which will generate the digital records of the student learning path. This article includes a bibliographic review of the role of ICT in the education processes and the fundamentals of the Flipped Classroom (FC) methodology. In the paper are included FC implementation experiences in higher education, followed by the presentation of the T-FliC Model as a technological proposal for this methodology. Finally, the conclusions present reflections on the proposal.

Keywords: Higher education; flipped classroom; technology model; ICT. 


\begin{abstract}
Resumo: Este trabalho apresenta uma proposta de modelo tecnológico para sala de aula invertida chamada T-FliC. O objetivo é dar um suporte, por meio das tecnologias da informação e comunicação (TIC), para o modelo pedagógico homônimo existente, a ser considerado em futuras implementações nas matérias de diferentes níveis de formação do ensino superior. T-FliC baseia-se principalmente no uso de recursos tecnológicos gratuitos, fundamentado em aplicações de Google (Sala de Aula, Drive e YouTube, entre outras), por causa de ser bastante utilizado por estudantes e professores, permitindo replicar este modelo em diferentes contextos educacionais. T-FliC incorpora cinco fases mediadas pelas TIC, que vão desde o planejamento das atividades de ensino-aprendizagem até a avaliação contínua das aprendizagens. Na sua implementação, o modelo T-FliC considera uma aula digital (aprendizagem fora da sala de aula) com orientação a sincrônica de um tutor virtual pessoal, uma oficina envolvendo o desenvolvimento de atividades dinâmicas para o trabalho colaborativo (aprendizagem em sala de aula), com orientação do tutor presencial e um processo de avaliação permanente e contínua mediada por ferramentas tecnológicas, como teclado, pasta e bloco que permite o registro digital do caminho de aprendizagem dos estudantes. Este artigo inclui uma revisão da literatura sobre o papel das TIC na educação e na base da metodologia da sala de aula invertida. São incorporados também os antecedentes de experiências de implementação de aula invertida no ensino superior, seguido da apresentação do modelo T-FliC como uma proposta tecnológica para essa metodologia. Finalmente, as reflexões sobre esta proposta encontram-se nas conclusões.
\end{abstract}

Palavras-chave: Ensino superior; aula invertida; modelo tecnológico; TIC.

\title{
Introducción
}

Existe acuerdo respecto a la importancia de la integración curricular de las tecnologías de la información y la comunicación (TIC) en educación superior (Adams Becker et al., 2017), particularmente en la generación de cambios sustanciales del proceso educativo en su conjunto. Es así como las TIC han promovido, no solo el cambio de rol de los distintos agentes educativos, sino además nuevas formas de interacción sincrónica y asincrónica, ampliando los espacios y tiempos de aprendizaje. En este sentido, la utilización de las tecnologías representa una oportunidad para realizar innovación educativa en las instituciones de educación superior. Este último aspecto implica la adopción de nuevas metodologías y la toma de decisiones pedagógicas en cuanto a las actividades didácticas y a la selección de los recursos tecnológicos a utilizar.

En este contexto, este artículo tiene por objetivo la presentación de una propuesta tecnológica para la implementación de Flipped Classroom (FC) o aula invertida como estrategia metodológica en asignaturas de distintos niveles de formación en educación superior. Este modelo tecnológico para Flipped Classroom denominado T-FliC tiene su sustento pedagógico en la propuesta realizada por Tourón y Santiago (2015) (Modelo pedagógico Flipped Classroom), en el cual el aprendizaje es el centro del proceso educativo, lo que es consistente con un modelo basado en competencias, con un estudiantado con un rol protagónico y un personal docente guía y facilitador del proceso de aprendizaje, mediante el uso de distintas metodologías activas que promuevan la integración de los distintos saberes del estudiantado y el desarrollo de sus habilidades cognitivas (De Miguel, 2005; Jerez, Hasbún y Rittershaussen, 2015). 
doi: http://dx.doi.org/10.15359/ree.22-2.2

URL: http://www.una.ac.cr/educare

CORREO: educare@una.cr

Esta propuesta tiene por objetivo entregar un soporte tecnológico para facilitar la implementación de FC, a partir de la utilización de las herramientas gratuitas de Google, las cuales, dado su uso masivo, permitirían replicar este modelo en cualquier área disciplinar en educación superior y en distintos contextos educativos.

\section{TIC y dimensiones asociadas al aprendizaje en educación superior}

En la sociedad actual, la formación de profesionales está caracterizada por un creciente acceso a la información, la relación con otros individuos y la generación de conocimiento, por lo cual las tecnologías de la información y comunicación (TIC) han pasado a jugar un rol fundamental no solo en el proceso de enseñanza-aprendizaje del estudiantado, sino también en el necesario desarrollo de sus habilidades digitales.

Ya desde fines del siglo XX ha habido iniciativas tendientes a establecer un marco de referencia para la educación superior ("Declaración de Bolonia", 1999) y concretar procesos de reforma curricular basados en competencias (Proyecto Tuning América Latina, 2007). En un modelo educativo basado en competencias, se debe entender el aprendizaje desde una perspectiva integradora que dinamice los conocimientos, habilidades y actitudes personales e interpersonales. En este sentido, adquiere relevancia la búsqueda de nuevas estrategias y metodologías de enseñanza, el seguimiento continuo de los aprendizajes logrados por el alumnado, flexibilidad en las modalidades de enseñanza y diversificación del sistema de evaluación (Ahumada, 2013; López, 2013; Villa y Poblete, 2007).

Antecedentes publicados por la CEPAL (Comisión Económica para América Latina y el Caribe) (Sunkel y Trucco, 2010) han permitido establecer una asociación positiva entre el uso de las TIC en el proceso educativo, la motivación y los logros académicos alcanzados por el estudiantado. De este modo, individuos motivados evidenciarán un alto compromiso emocional y conductual con la tarea asignada, lo que se traducirá en un buen rendimiento académico (Claro, 2010).

\section{Flipped classroom: Conceptos y fundamentos}

Para Lage y Platt (2000) y Lage, Platt y Treglia (2000), Flipped classroom (FC) no es otra cosa que "clases invertidas", entendiendo, por esto, que lo que tradicionalmente se enseñaba dentro del aula ahora se aprende fuera de ella, lo cual implica solo una reorganización de las actividades en función del lugar donde se ejecutan. Sin embargo, trabajos actuales (Bergmann y Sams, 2012; Bishop y Verleger, 2013; McDonald y Smith, 2013; Tourón y Santiago, 2015; Tucker, 2012) consideran a FC como un modelo pedagógico que incluye actividades de aprendizaje interactivas y grupales dentro del aula, ya que utilizan este tiempo para potenciar procesos de adquisición y práctica de conocimientos teóricos, y transferir el trabajo individual de aprendizaje fuera del aula. 
Para Bergmann y Sams (2012), FC tiene un enfoque integral que conecta la instrucción directa con métodos constructivistas, permitiendo que el estudiantado comprenda la información, la analice y aplique, por lo tanto, propicia el desarrollo y manejo de sus habilidades cognitivas. Este último aspecto resalta la importancia que tiene el diseño de actividades que fomenten el autoaprendizaje o enfocadas en el trabajo autónomo del estudiantado, ya que promueven competencias de naturaleza metacognitiva (Fernández et al., 2013; López, 2013).

Se han documentado evidencias acerca de cómo la utilización de FC en elámbito educativo favorece el proceso de enseñanza-aprendizaje. En el Reporte del Observatorio de Innovación Educativa del Tecnológico de Monterrey (2014) se presentan resultados y experiencias de parte de su profesorado. Este resalta lo positivo del ambiente de trabajo en aula al desarrollar actividades más significativas, el aumento en la motivación del alumnado y un mayor compromiso con su proceso educativo. Asimismo, indica que se evidencian mejores resultados de aprendizaje alcanzados por el estudiantado.

Los resultados de aprendizaje y la motivación del estudiantado hacia el uso de las TIC en su proceso de aprendizaje son variables y deben medirse para evaluar el efecto de la incorporación de la metodología Flipped classroom (FC), por lo tanto, tienen que ser consideradas al momento del diseño de dicha implementación. Adicional a lo anteriormente señalado, deben incorporarse algunos modelos de aprendizaje que le den sustento teórico a la propuesta, dentro de los cuales destaca la teoría del constructivismo social de Vygotsky (en Woolfolk, 2010; Gutiérrez y Rada, 2012), el conectivismo (Siemens, 2004; Zapata-Ros, 2015) y el modelo FC (Tourón y Santiago, 2015).

Para Vygotsky, el proceso de aprendizaje individual y de construcción del conocimiento se basa en la internalización del individuo de los aprendizajes desarrollados a partir de la interacción con otros (Woolfolk, 2010). En este sentido, el constructivismo social, considera el conocimiento como el resultado de la relación entre el individuo y su medio sociocultural (Gutiérrez y Rada, 2012).

Al analizar la dimensión del aprendizaje de Vygotsky, a partir de mi relación con otras personas, inevitablemente debemos considerar el rol que juegan hoy las tecnologías y cómo han sido incorporadas a las actividades de enseñanza aprendizaje en distintos contextos educativos. Desde esta perspectiva, el conectivismo hace énfasis no solo en la generación de conocimiento, sino en la transferencia de este a través de la actividad social que se genera entre los individuos conectados, con lo cual se logran aprendizajes a partir de las experiencias e interacciones con otros seres (Downes, 2012; Siemens, 2004).

El conectivismo considera la presencia relevante de las TIC y las relaciones que se crean de su uso. En este sentido, Downes (2012) introduce un concepto definido como la teoría conectivista del aprendizaje en línea, que concibe el aprendizaje como un proceso en red, hace especial énfasis en que el conocimiento está en las conexiones entre las personas y que el 
doi: http://dx.doi.org/10.15359/ree.22-2.2

URL: http://www.una.ac.cr/educare

CORREO: educare@una.cr

aprendizaje se genera a partir del cruce de estas conexiones. Se valora no solo la acumulación de información disponible en la red o la tecnología, sino también la capacidad de saber qué hacer con esa información.

Si consideramos ambos modelos teóricos, no hay que olvidar que la educación actual debe entender la tecnología como un recurso y no como un fin, y que su incorporación debe promover el aprendizaje activo y colaborativo, con estudiantes responsables en su proceso educativo y con una evaluación continua y permanente con énfasis en la retroalimentación oportuna. Estos aspectos claves son los que sustentan el modelo pedagógico Flipped classroom (FC) (Bergmann y Sams, 2012; Bishop y Verleger, 2013; McDonald y Smith, 2013; Tourón y Santiago, 2015; Tucker, 2012), en el cual el aprendizaje es el centro del proceso educativo, con estudiantes con un rol protagónico y docentes como guías cuya función es facilitar el proceso formativo.

Si bien no existe una guía única para implementar FC, Hamdan, McKnight, McKnight y Arfstrom (2013) han podido identificar cuatro pilares que deben considerarse en su implementación:

- Ambientes flexibles: el estudiantado puede elegir cuándo y dónde aprenden, es decir, el aprendizaje no está limitado a un horario y a un espacio físico determinado (aprendizaje ubicuo) (Burbules, 2012).

- Cultura de aprendizaje: se basa en un modelo educativo centrado en el estudiantado, en el cual se prioriza el tiempo en aula para desarrollar actividades dinámicas que posibiliten profundizar el conocimiento y desarrollar en el estudiantado niveles de pensamiento superior. Se valora positivamente la retroalimentación permanente y oportuna por la parte docente.

- Contenido intencional: es relevante establecer los resultados de aprendizaje esperados, para generar un diseño con las temáticas a abordar y actividades que serán realizadas en aula o de forma autónoma por parte del estudiantado

- Docente profesional: se requiere un personal docente flexible, dispuesto a establecer mejoras en el proceso de enseñanza-aprendizaje, con un rol de guía y facilitador; planificado, es decir, que seleccione recursos o metodologías de trabajo teniendo presente los resultados de aprendizaje esperados, el tiempo efectivo a utilizar en las diferentes actividades dentro y fuera del aula, y el tipo de instrumentos evaluativos que permitan monitorear los logros alcanzados por el alumnado, con ello hará la práctica docente más efectiva y eficiente. 


\section{Experiencias de implementación de Flipped classroom}

En relación con el impacto de la aplicación de FC en estudiantado de educación superior, se ha observado una mejora en su rendimiento académico (Chiple y Ramos, 2014; Hasbún, 2014; Sengel, 2016). Para Bishop y Verleger (2013) el que FC se fundamente en el aprendizaje centrado en el estudiantado y el uso de metodologías activas es suficiente evidencia de que este logre un aprendizaje más significativo.

Recientes investigaciones han demostrado la preferencia del estudiantado por esta metodología en contraposición a la clase tradicional, ya que están más involucrados, motivados y comprometidos en el proceso de aprendizaje. También logran evidenciar mejoras en el aula debido a las actividades que propician el aprendizaje activo con uso de diferentes recursos tecnológicos (Chen, Yang y Hsiao, 2016; Sengel, 2016).

Long, Cummins y Waugh (2017), por medio de un estudio cualitativo realizado a profesorado de diversas disciplinas académicas y nivel de experiencia en esta metodología, se percataron de una valoración positiva respecto a la optimización del tiempo en el aula donde estudiantes aplican los contenidos previos en las actividades desarrolladas en el aula, así como también, una mayor motivación y compromiso de docentes

En cuanto a los diferentes recursos tecnológicos usados para implementar FC, Zainuddin y Halili (2016), a través de una revisión bibliográfica, destacan el uso de videos de YouTube y de Khan Academy, WebQuest, Dropbox y herramientas de Google, las que propician más oportunidades de interacción entre estudiantes tanto fuera como dentro del aula. Estos autores destacan la importancia de la capacitación de docentes en esta metodología y sugieren la creación de videos que incorporen elementos como animación, caricatura y música para lograr captar la atención del estudiantado. Finalmente, concluyen que el uso de FC abarca los diferentes estilos de aprendizaje, lo que conduce a un proceso formativo efectivo, activo y que desarrolla habilidades de pensamiento de orden superior.

\section{Modelo tecnológico para Flipped classroom (T-FliC)}

Este artículo propone un modelo tecnológico para Flipped Classroom (T-FliC) (ver figura 1) cuyo objetivo es dar un soporte mediado por las TIC al modelo pedagógico propuesto por Tourón y Santiago (2015). Cabe mencionar que la puesta en marcha del modelo T-FliC debe incorporar, por parte del personal docente, la revisión de las temáticas, los resultados de aprendizaje de la asignatura en cuestión y una adecuada planificación de las acciones pedagógicas y así asegurar el uso de los recursos tecnológicos en el contexto curricular. 
doi: http://dx.doi.org/10.15359/ree.22-2.2

URL: http://www.una.ac.cr/educare

CORREO: educare@una.cr

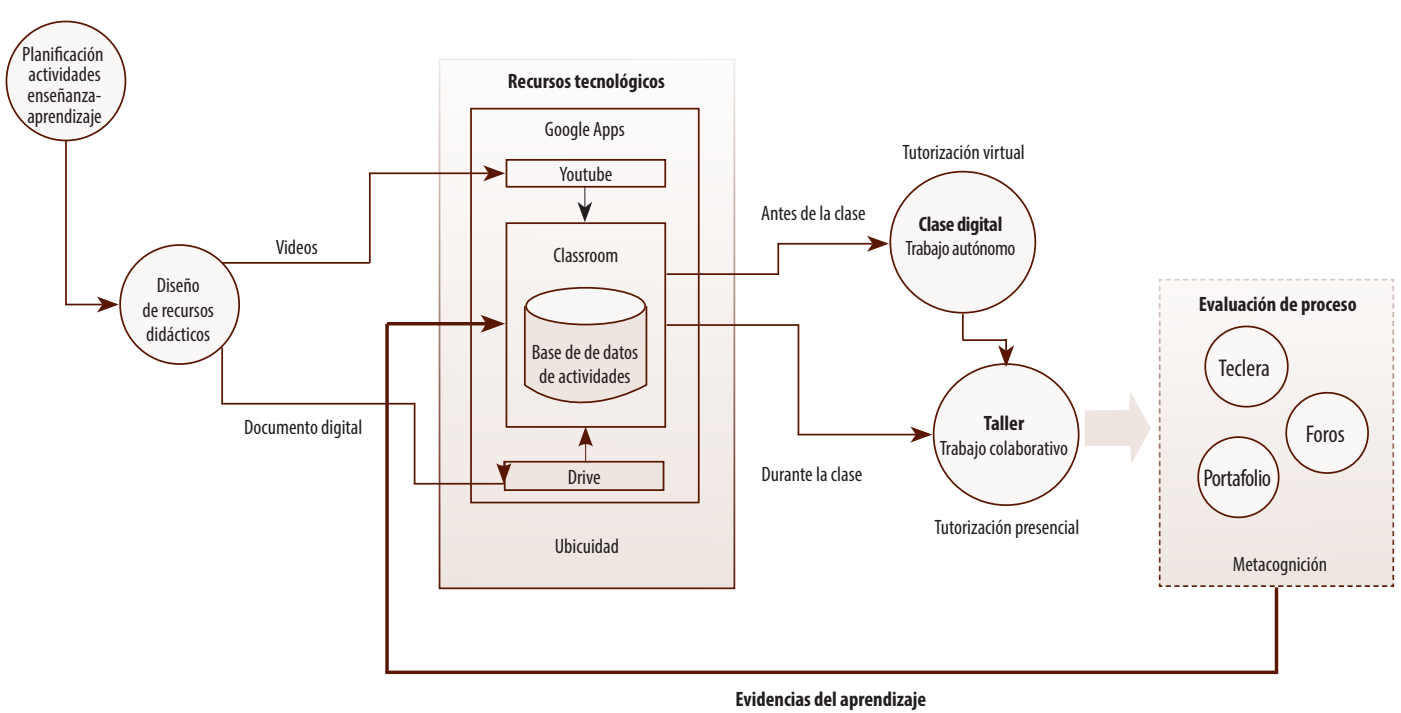

Figura 1: Modelo tecnológico para Flipped Classroom.

Nota: Elaboración propia.

El Modelo T-FliC propuesto se basa en la utilización de aplicaciones Google como fuente de los principales recursos tecnológicos para la implementación de Flipped Classroom.

En el ámbito de los medios tecnológicos se propone utilizar estas aplicaciones de Google (Google Apps) debido a su amplio espectro de recursos digitales ofrecidos, los cuales son de uso masivo por un gran número de estudiantes y docentes, en distintos ámbitos educativos. Dentro de los recursos de Google se puede mencionar: Classroom, Drive, Youtube, Google Docs, entre otros. Por su carácter de gratuidad, posibilita que cualquier institución educativa disponga de este servicio sin incurrir en un gasto de implementación de plataformas virtuales de aprendizaje y servicios anexos que esto conlleva, remitiéndose solo a contar con una conexión de internet.

\section{Funcionamiento de las herramientas:}

- Classroom de Google como plataforma virtual de aprendizaje, en la que se dispondrá todo el material pedagógico necesario para el desarrollo de las actividades de aprendizaje declaradas en la asignatura, estos son: los recursos audiovisuales y documentos digitales como material de apoyo para el trabajo autónomo y colaborativo. Esta herramienta se usará como base de datos para registrar las actividades que desarrolle el estudiantado para almacenar las evidencias de sus avances y logros con los aportes individuales y grupales. 
- Youtube como soporte de los videos, los cuales contienen el material audiovisual previamente preparado por cada docente y que serán compartidos a través del canal privado asociado a la asignatura respectiva y que estarán a disposición del estudiantado por medio de la plataforma Classroom.

- Drive como medio digital de almacenamiento de la documentación complementaria para el estudiantado y repositorio de los documentos que se soliciten a participantes para trabajo individual o colaborativo.

- Documentos, hojas de cálculo y presentaciones de Google Docs, como herramientas de ofimática para el desarrollo de documentos escritos, presentaciones y sociabilización de los trabajos, para promover el aprendizaje colaborativo y la gestión del conocimiento (Careaga, 2008; Careaga y Avendaño, 2015).

\section{Etapas del modelo}

\section{Planificación de actividades de enseñanza-aprendizaje}

En esta fase el personal docente planifica, de acuerdo con los resultados de aprendizaje esperados declarados en la asignatura, el conjunto de recursos didácticos necesarios para las actividades que se quieran desarrollar en la asignatura, tanto para el trabajo autónomo del estudiantado (videos, apuntes, guías de ejercicios u otros) como para el quehacer al interior del aula (actividades de aprendizaje activo).

Para la preparación de los videos se deben considerar varios aspectos relevantes como son los contenidos a desarrollar, guion, duración máxima, calidad de imagen y sonido, tiempo de edición y, sobretodo, cómo se acoplan estos a las actividades de aula, lo que conlleva a la apropiación de las temáticas analizadas.

Como resultado de esta etapa se obtendrá el syllabus o programación del curso, que incorpora no solo los contenidos y resultados de aprendizaje sino también las metodologías, actividades, recursos, y las estrategias evaluativas que permitan monitorear los avances y logros alcanzados por el estudiantado (Jerez et al., 2015).

\section{Diseño de recursos didácticos}

Etapa en la cual el personal docente con el grupo de apoyo técnico (personal de diseño gráfico, docentes del área, entre otro) procede a grabar los videos con las temáticas previamente seleccionadas y según la planificación establecida. Considerando el amplio espectro de herramientas tanto gratuitas como de pago, la estrategia dependerá de los recursos con que 
doi: http://dx.doi.org/10.15359/ree.22-2.2

URL: http://www.una.ac.cr/educare

CORREO: educare@una.cr

disponga la institución para esta actividad. Se sugiere Screencast ${ }^{5}$ y Camtasia $^{6}$, entre otros. Se considera, además, la selección de material digital (artículos científicos, infografías, apuntes, guías de ejercicios, entre otros) que el docente estime conveniente como lectura fundamental o complementaria en concordancia con los contenidos establecidos y que servirán de apoyo al estudiantado en su proceso de aprendizaje autónomo.

Se obtendrá de esta fase el almacenamiento de los recursos audiovisuales en un canal privado de Youtube y un repositorio de las actividades didácticas y del material complementario seleccionado, los cuales serán almacenados en Drive.

\section{Clase digital}

Fase que comprende los recursos electrónicos didácticos que el estudiantado debe revisar, en forma virtual, antes de cada sesión presencial. En este sentido y de acuerdo con las consideraciones de la metodología Flipped Classroom, se asigna un rol protagónico al estudiante, mediante el traspaso de la responsabilidad individual de revisar los contenidos teóricos que se encuentran disponibles digitalmente en la plataforma, a través de los vídeos y del material complementario.

Antes de la realización de cada sesión presencial, se sugiere levantar en el foro de Classroom, preguntas o afirmaciones asociadas al contenido para potenciar la reflexión y la metacognición del estudiantado (Iglesias y Suárez, 2003; Osses y Jaramillo, 2008).

En esta etapa, se considera un profesor o profesora auxiliar o personal tutor virtual para dar dinamismo a la comunidad educativa en cuanto a monitorear la revisión del material preparado, la participación en los foros y realizar una retroalimentación oportuna asincrónica (tutorización virtual). Las funciones de este personal tutor virtual serán, principalmente, técnica (gestión y apoyo del entorno virtual de aprendizaje) y de orientación (asesoramiento personalizado), para garantizar el desarrollo de la acción formativa (Cabero, Llorente y Gisbert, 2007; Silva y Astudillo, 2013).

\section{Taller}

Esta etapa corresponde a la sesión presencial realizada en el aula, en la cual el estudiantado desarrolla actividades de aprendizaje con metodologías activas (aprendizaje basado en proyectos, aprendizaje basado en problemas, entre otros) con la modalidad de trabajo en equipo (De Miguel, 2005; López, 2013). El objetivo de esta fase es que el alumnado sea capaz de aplicar los contenidos aprendidos virtualmente, mediante los recursos disponibles en la plataforma

\footnotetext{
${ }^{5}$ Camtasia es localizable en https://screencast-o-matic.com/home

${ }^{6}$ Camtasia es localizable en http://discover.techsmith.com/camtasia-brand-desktop/
} 
Classroom, con la mediación docente (tutorización presencial) y en colaboración con sus pares, lo cual propicia un aprendizaje significativo (Osses y Jaramillo, 2008).

Como resultado de esta etapa, se obtendrán las evidencias del trabajo colaborativo (fotografías, videos, entre otros) realizado en el aula y de los productos desarrollados por el estudiantado, los cuales se traducirán en documentos digitales subidos y compartidos desde el recurso Drive.

Se sugiere que el personal docente o tutor presencial lleve registro de cada sesión, el desarrollo de las actividades, nivel de cumplimiento de las metas, desempeño del estudiantado, real pertinencia de las actividades según las temáticas, entre otros, para realizar los ajustes y mejoras, si se requiriera en una próxima implementación.

\section{Evaluación de proceso}

Fase en la cual se realizará el levantamiento continuo y sistemático de datos de todo el proceso de aprendizaje del estudiantado, queda en evidencia el nivel de avance y logro alcanzado respecto a los aprendizajes esperados en la asignatura o unidad temática. Este tipo de evaluación en tiempo real posibilita la reflexión permanente por parte del estudiantado acerca de su proceso formativo y al personal docente respecto a su práctica, con lo que se propicia la toma de decisiones de ajustes y mejoras, si se requieren (Barberà, 2006).

Esta etapa se considera clave en el proceso educativoado ya que facilita que el estudiante tome conciencia acerca de lo que aprendió, cómo lo hizo y para qué lo hizo, de modo que esto le permita la toma de decisiones para la solución de diferentes situaciones problemas.

Para el desarrollo de esta fase se considera la utilización de recursos tecnológicos pertinentes, dentro ${ }^{7}$ de los cuales se sugiere la incorporación de tecleras o similares (Socrative ${ }^{8}$, Kahoot y Nearpod ${ }^{9}$ ), las cuales, a través de sus aplicaciones móviles, posibilitan la realización de evaluaciones in-situ, con la incorporación de los celulares y tabletas del propio estudiantado, como herramientas innovadoras en el proceso evaluativo.

Adicionalmente, se sugiere levantar preguntas reflexivas a través del foro de Classroom, los cuales serán guiados por el personal tutor virtual, para favorecer que las a portaciones realizadas por el estudiantado contribuyan al aprendizaje social.

\footnotetext{
${ }^{7}$ Kahoot es localizable en https://kahoot.com/

${ }^{8}$ Socrative es localizable en http://www.socrative.com/

${ }^{9}$ Nearpod es localizable en https://nearpod.com/
} 
doi: http://dx.doi.org/10.15359/ree.22-2.2

URL: http://www.una.ac.cr/educare

CORREO: educare@una.cr

Como producto de esta etapa se considera un portafolio creado en Classroom, cuyo objetivo será tener el registro digital de la ruta de aprendizaje de cada estudiante, la cual quedará evidenciada, por ejemplo, en los reportes de desempeño que entregan las aplicaciones como Socrative, en el porcentaje de participación y pertinencia de las contribuciones realizadas en los foros $y$ en el material generado por el estudiantado en el desarrollo de las actividades del taller.

\section{Conclusiones}

El modeloT-FliC es una propuesta tecnológica para la implementación de Flipped Classroom en asignaturas de distintos niveles de formación en educación superior, la cual se sustenta en la incorporación de recursos digitales gratuitos (por ejemplo, aplicaciones de Google), para posibilitar que la experiencia pueda ser replicada en cualquier institución de educación que quiera innovar en su proceso de enseñanza-aprendizaje, independiente de su presupuesto para inversión en tecnología.

Se destaca la utilización de las herramientas disponibles en Google como un componente tecnológico diferenciador, ya que, debido a su gratuidad y uso masivo tanto por docentes como estudiantes, en el ámbito académico y personal, facilita la implementación del modelo en distintos contextos educativos. En concordancia con lo anterior, Zainuddin y Halili (2016) distinguen las herramientas de Google dentro del espectro de medios tecnológicos utilizados en esta metodología. Además, cabe señalar que el carácter universal de estos recursos posibilita su fácil apropiación por parte de todos los actores participantes del proceso educativo.

TFliC aporta los lineamientos generales para la implementación tecnológica de FC, entregando al personal docente un set de herramientas de Google que puede seleccionar y utilizar, según el tipo de actividad didáctica planificada.

Adicionalmente, una ventaja del uso de las herramientas tecnológicas consideradas en TFliC es propiciar la interrelación de comunidades docentes que puedan compartir sus experiencias en un contexto de educación superior. En este sentido, estos recursos tecnológicos de carácter universal permitirían ampliar el espacio físico hacia un espacio virtual que favorezca la comunicación entre distintas comunidades docentes tanto a nivel nacional como internacional.

En el modelo T-FliC se incorporan cinco etapas mediadas por las TIC, que van desde la planificación de las actividades de enseñanza-aprendizaje, hasta la evaluación continua de los aprendizajes logrados y los que están en proceso de ser logrados. Es importante indicar que, en el desarrollo de estas fases, se deben considerar, necesariamente, los resultados de aprendizajes esperados y las competencias declaradas en los programas de las asignaturas, de modo que exista coherencia y pertinencia entre las actividades diseñadas y las metas propuestas. 
El modelo T-FliC considera, como base, los aspectos planteados por Tourón y Santiago (2015) y Hamdan et al. (2013) en cuanto a los principales elementos de FC, es decir, incorpora:

- Un ambiente flexible de aprendizaje, a través de videos en youtube y material de apoyo en Classroom de Google, que propician el trabajo autónomo del estudiantado en cualquier momento y lugar (aprendizaje fuera del aula).

- Un modelo educativo centrado en el estudiantado, con docentes como guías (personal tutor presencial) del proceso de aprendizaje y a través del desarrollo de actividades dinámicas para el trabajo colaborativo en aula, muchas de las cuales serán mediadas por las TIC y cuyas evidencias serán almacenadas en Drive.

- Retroalimentación permanente y oportuna, a través de evaluaciones formativas con TIC y de la comunicación asincrónica en foros guiados por personal de tutoría virtual.

- Un proceso evaluativo, entendido como un sondeo permanente que permita monitorear los avances y logros alcanzados por el alumnado, con un marcado énfasis en la reflexión.

Por otra parte, se favorece la interacción entre los distintos agentes educativos del proceso formativo, al considerar los diversos mecanismos de evaluación presentes en el modelo T-FliC que propician la interrelación entre estudiante-estudiante, estudiante-docente y docente-estudiante en el fomento de la metacognición. Lo anterior es coherente con lo planteado por Downes (2012) en cuanto a que el aprendizaje se concreta a partir del cruce de las conexiones en red de los individuos.

Como trabajo a futuro se propone pilotear el modelo T-FliC en instituciones de educación superior, con el objetivo de evaluar su implementación y establecer ajustes, si fueran necesarios. Esta evaluación deberá considerar aspectos como calidad y pertinencia de los recursos digitales utilizados, participación virtual de estudiantes en forma individual y en su trabajo colaborativo, accesibilidad a la plataforma Classroom y calidad de las evidencias digitales de los aprendizajes estudiantiles. En este sentido, adquiere relevancia una adecuada capacitación a docentes que considere las dimensiones pedagógica y tecnológica de Flipped Classroom.

\section{Referencias}

Adams-Becker, S., Cummins, M., Davis, A., Freeman, A., Hall Giesinger, C. y Ananthanarayanan, V. (2017). NMC Horizon Report: 2017 Higher Education Edition. Austin, Texas: The New Media Consortium. Recuperado de http://cdn.nmc.org/media/2017-nmc-horizon-report-he-EN.pdf

Ahumada, M. E. (2013). Las TIC en la formación basada en competencias. Revista Universidad de La Salle, 60, 141-157. Recuperado de https://revistas.lasalle.edu.co/index.php/ls/article/ view/2388/2133 
doi: http://dx.doi.org/10.15359/ree.22-2.2

URL: http://www.una.ac.cr/educare

CORREO: educare@una.cr

Barberá, E. (2006). Aportaciones de la tecnología a la e-Evaluación. RED: Revista de Educación a Distancia. Monograph 6, 1-13. Recuperado de http://www.um.es/ead/red/M6/barbera.pdf

Bergmann, J. y Sams, A. (2012). Flip your classroom: Reach every student in every class every day. United States of America: International Society for Technology in Education. Recuperado de https://www.liceopalmieri.gov.it/wp-content/uploads/2016/11/Flip-Your-Classroom.pdf

Bishop, J. y Verleger, M. (June, 2013). The flipped classroom: A survey of the research. In 120th ASEE National Conference \& Exposition. Atlanta: American Society for Engineering Education. Recuperado de https://www.asee.org/public/conferences/20/papers/6219/view

Burbules, N. (2012). El aprendizaje ubicuo y el futuro de la enseñanza. Encounters on Education, 13, 3-14. Recuperado de https://dialnet.unirioja.es/ejemplar/318240

Cabero, J., Llorente, M. y Gisbert, M. (2007). El papel del profesor y el alumno en los nuevos entornos tecnológicos de formación. En J. Cabero (Coord.), Nuevas tecnologías aplicadas a la educación (pp. 261-277). Madrid: McGraw-Hill.

Careaga, M. (2008). Gestión del conocimiento. "Modelos dereferencia para su inserción en Educación" (Parte 2) [Mensaje en un blog]. Recuperado de http://www.eduglobal.cl/2013/09/13/ gestion-del-conocimiento-modelos-de-referencia-para-su-insercion-en-educacion/

Careaga, M. y Avendaño, A. (2015). Currículum cibernético y gestión del conocimiento. Fundamentos y modelos de referencia. Chile: Editorial UCSC.

Chen, S.-C., Yang, S. J. H. y Hsiao, C-C. (2016). Exploring student perceptions, learning outcome and gender differences in a flipped mathematics course. British Journal of Educational Technology, 47(6), 1096-1112. doi: https://doi.org/10.1111/bjet.12278

Chiple, R.y Ramos, A. (2014). Acompañamiento 100\% presencial para una modalidad 100\% virtual. En P. Requeijo y C. Gaona (Eds.), Contenidos innovadores en la universidad actual (pp. 203218). Madrid: McGraw-Hill. Recuperado de https://www.researchgate.net/profile/Jose Diaz-Cuesta/publication/280551145 Masculinidades que dejan huella en \%27The Sugarland Express\%27 Spielberg 1974/links/55b8a07b08aed621de05f19f/ Masculinidades-que-dejan-huella-en-The-Sugarland-Express-Spielberg-1974.pdf

Claro, M. (2010). Impacto de las TIC en los aprendizajes de los estudiantes: Estado del arte. Santiago de Chile: Naciones Unidas. Recuperado de http://repositorio.cepal.org/bitstream/ handle/11362/3781/lcw339.pdf? sequence=1\&isAllowed $=y$ 
De Miguel, M. (Dir.). (2005). Modalidades de enseñanza centradas en el desarrollo de competencias. Orientaciones para promoverel cambio metodológico en el espacio europeo de educación superior. Oviedo: Ediorial Universidad de Oviedo. Recuperado de https://www. upc.edu/rima/ca/grups/grapa/bibliografia-evaluacion/publicaciones/modalidades-deensenanza/view

Declaración de Bolonia. Declaración conjunta de los ministros europeos de educación. (1999). Recuperado de http://www.educacion.gob.es/boloniaensecundaria/img/Declaracion Bolonia.pdf

Downes, S. (2012). Connectivism and connective knowledge. Essays on meaning and learning networks. Canadá: National Research Council Canada. Recuperado de https://pdfs. semanticscholar.org/4718/ee3c1930820e094552f0933cbc3b86548dbc.pdf

Fernández, E., Bernardo, A., Suárez, N., Cerezo, R., Núñez, J. y Rosário, P. (2013). Predicción del uso de estrategias de autorregulación en educación superior. Anales de psicología, 29(3), 865875. doi: https://doi.org/10.6018/analesps.29.3.139341

Gutiérrez, I. y Rada, C. (2012). El pensamiento constructivista como ideal en la universidad. Arte \& Diseño, 10(2), 23-27. Recuperado de http://ojs.uac.edu.co/index.php/arte-diseno/article/ view/127/112

Hamdan, N., McKnight, P., McKnight, K. y Arfstrom, K. (2013). The flipped learning model: A white paper based on the literature review titled A review of flipped learning. Arlington, VA: Flipped Learning Network.

Hasbún, B. (2014). Flipped Classroom en FEN U de Chile [Presentación en Prezi]. Recuperado de https://prezi.com/hur jd zom3f/flipped-classroom-en-fen-u-de-chile/

Iglesias, R. R. y Suárez, C. O. (2003). El papel de la metacognición en la sistematización de las habilidades cognitivas. Psicología, 101, 126-130.

Jerez, O., Hasbún, B. y Rittershaussen, S. (2015). El diseño de Syllabus en la educación superior. Una propuesta metodológica. Chile: Ediciones Universidad de Chile. Recuperado de http://goo. $\mathrm{gl} / \mathrm{SgFsc} 4$

Lage, M. J. y Platt, G. J. (2000). The internet and the inverted classroom. The Journal of Economic Education, 31(1), 11-11. Doi: https://doi.org/10.1080/00220480009596756

Lage, M. J., Platt, G. J. y Treglia, M. (2000). Inverting the classroom: A gateway to creating an inclusive learning environment. The Journal of Economic Education, 31(1), 30-43. doi: https://doi.org/10.2307/1183338 
doi: http://dx.doi.org/10.15359/ree.22-2.2

URL: http://www.una.ac.cr/educare

CORREO: educare@una.cr

Long, T., Cummins, J. y Waugh, M. (2017). Use of the flipped classroom instructional model in higher education: Instructors' perspectives. Journal of Computing in Higher Education, 29(2), 179-200. doi: https://doi.org/10.1007/s12528-016-9119-8

López, M. Á. (2013). Aprendizajes, competencias y TIC. México: Pearson.

McDonald, K. M. y Smith, C. M. (2013). The flipped classroom for professional development: Part I. Benefits and strategies. The Journal of Continuing Education in Nursing, 44(10), 437-438. doi: https://doi.org/10.3928/00220124-20130925-19

Observatorio de Innovación Educativa del Tecnológico de Monterrey (2014). Aprendizaje Invertido. Reporte Edutrends, 1-27. Recuperado de http://goo.gl/aFiD55

Osses, S. y Jaramillo, S. (2008). Metacognición: Un camino para aprender a aprender. Estudios pedagógicos, 34(1), 187-197. doi: https://doi.org/10.4067/S0718-07052008000100011

Proyecto Tuning América Latina (2007). Reflexiones y perspectivas de la educación superior en América Latina. Informe final Proyecto Tuning América Latina, 2004-2007. Bilbao: Publicaciones de la Universidad de Deusto. Recuperado de http://goo.gl/D8EbzO

Sengel, E. (2016). To FLIP or not to FLIP: Comparative case study in higher education in Turkey. Computers in Human Behavior, 64, 547-555. Recuperado de https://doi.org/10.1016/j. chb.2016.07.034

Siemens, G. (2004). Conectivismo: Una teoría de aprendizaje para la era digital (Trad. por D. Leal Fonseca).

Silva, J. E. y Astudillo, A. (2013). Formación de tutores: Aspecto clave en enseñanza virtual. Didasc@lia: Didáctica y Educación, 4(1), 87-100. Recuperado de https://dialnet.unirioja.es/ servlet/articulo?codigo $=4233643$

Sunkel, G. y Trucco, D. (2010). Nuevas tecnologías de la información y la comunicación para la educación en América Latina: Riesgos y oportunidades. Santiago de Chile: Naciones Unidas. Recuperado de http://repositorio.cepal.org/bitstream/handle/11362/6174/lcl3266. pdf? sequence $=1$ \&isAllowed $=y$

Tourón, J. y Santiago, R. (2015). Flipped learning model and the development of talent at school. Revista de Educación, 368, 33-65. doi: 10.4438/1988-592X-RE-2015-368-288

Tucker, B. (2012). The flipped classroom. Education Next, 12(1), 82-83. Recuperado de http:// educationnext.org/the-flipped-classroom/ 
doi: http://dx.doi.org/10.15359/ree.22-2.2

URL: http://www.una.ac.cr/educare

Villa, A. y Poblete, M. (Dirs.). (2007). Aprendizaje basado en competencias: Una propuesta para la evaluación de las competencias genéricas. Bilbao: Ediciones Mensajero. Recuperado de http://biblio.upmx.mx/textos/14633.pdf

Woolfolk, A. (2010). Psicología educativa (11 a ed.). México: Pearson. Recuperado de https:// crecerpsi.files.wordpress.com/2014/03/libro-psicologia-educativa.pdf

Zainuddin, Z. y Halili, S. H. (2016). Flipped classroom research and trends from different fields of study. International Review of research in Open and Distributed Learning, 17(3), 313-340. doi: https://doi.org/10.19173/irrodl.v17i3.2274

Zapata-Ros, M. (2015). Teorías y modelos sobre el aprendizaje en entornos conectados y ubicuos: Bases para un nuevo modelo teórico a partir de una visión crítica del" conectivismo". Education in the Knowledge Society (EKS), 16(1), 69-102. doi: https://doi.org/10.14201/ eks201516169102 